\title{
VI. - QUALITÉ DU MAḮS DESTINÉ A L'AMIDONNERIE
}

\author{
L. SAINT-LÈBE \\ Service de Radioagronomie, Département de Biologie, \\ Centre d'Études nucléaires de Cadarache, 13 - Saint-Paul-lèz-Durance

\section{RÉSUMÉ}

Les analyses commerciales ne révèlent pas dans tous les cas la dépréciation réelle de la qualité du maïs destiné à l'amidonnerie, alors que l'étude du rendement en amidon et de la rhéologie des empois permettent de suivre l'évolution de la valeur amidonnière du grain. Le séchage industriel avec de l'air à $80^{\circ} \mathrm{C}$ est sans effet sur le rendement en amidon et modifie peu les caractéristiques rhéologiques. Par contre, le grain est profondément dégradé et l'amidon altéré lorsque la température atteint ${ }^{1} 4^{\circ}{ }^{\circ} \mathrm{C}:$ le mais obtenu dans ces conditions constitue une matière première de mauvaise qualité pour l'amidonnerie.

\section{IN'TRODUCTION}

L,e maïs est devent, depuis quelques années, une matière première industrielle importante : en I968-69, les amidonneries françaises ont traité 500 ooo tonnes de cette céréale, soit 1'équivalent de I2 à I 5 p. Ioo de la collecte nationale, selon une étude de 1'Association pour la Promotion Industrie-Agriculture (A. P. R. I. A., I968). Les critères de qualité retenus dans l'industrie amidonnière sont différents de ceux qui sont appliqués par les organismes chargés d'assurer le stockage et la vente des céréales. Il s'agit surtout de la quantité d'amidon extractible industriellement et de sa viscosité à l'empesage.

Cette étude a pour but de rechercher les corrélations existant entre les deux critères de qualité précédents, les analyses commerciales, et les nombreux tests physiques et biochimiques appliqués sur les échantillons représentatifs de traitements effectués à une échelle semi-industrielle (LASSERAN, I).

Des travaux similaires entrepris sur des maïs séchés à différentes températures, mais au laboratoire, ont montré que les grains ainsi obtenus constituaient pour les industriels une matière première de qualité médiocre (GAUsmaN et al., I952, Mc 
MASTERs et al., I954; Mc MASTERs et al., I959; Watson et Hirata, I962); la démarche la plus exhaustive étant celle de WAHL, (WAHL, I966, $a$ et $b$ ) qui examine à la fois l'influence de la température de l'air et du rythme du séchage en fonction de l'humidité initiale et finale du grain.

\section{MATÉRIEI, ET MÉTHODES}

Il n'y a aucune relation entre la quantité d'amidon déterminée à partir de dosages physiques ou chimiques et celle qui est extraite dans l'industrie. Afin de définir cette dernière valeur, une méthode qui est l'adaptation à l'échelle du laboratoire du processus industriel a été mise au point (SAINT-LèBE et al., I965); elle permet à partir d'un échantillon de $200 \mathrm{~g}$ de mais de définir un taux d'extraction comparable à celui que l'on obtient en amidonnerie : 64 à $7 \circ \mathrm{p}$. Ioo $\pm 0,4$.

L'étude rhéologique a été réalisée à l'aide d'un viscographe BRABENDER. Une suspension de $22 \mathrm{~g}$ d'amidon sec dans $45^{\circ} \mathrm{ml}$ d'eau est chauffée régulièrement de $\mathrm{I}, 4^{\circ} \mathrm{C}$ par minute jusqu'à $96^{\circ} \mathrm{C}$, maintenue à cette température pendant 30 minutes puis refroidie selon le même rythme.

Les analyses ont été effectuées sur des grains précédemment triés et exempts de brisures.

\section{RÉSULTATS}

\section{I. - Rendement en amidon}

Les conclusions que l'on peut déduire des résultats obtenus (tabl. I) sont les suivantes :

— Le témoin récolte (TR) n'est pas significativement différent des témoins de traitement (stade I : TTR, TTE, TTF). Le séchage des grains avec de l'air ambiant ou très peu chauffé est donc sans influence, comparé au même séchage appliqué aux épis récoltés manuellement. Par contre, il n'est pas possible de conclure quant à l'incidence du mode de récolte, manuel ou mécanique, puisque les grains cassés ont été éliminés avant analyse.

- Le préstockage du maïs humide (Stade II) avec ventilation d'air refroidi (échantillons $\mathrm{F}_{0}$ ) a un effet dépressif significatif $(2,6 \mathrm{p}$. Ioo en valeur relative); il en est de même pour un échauffement naturel en cellule (échantillons $E_{0}$ ), mais dans ce cas la diminution de rendement est environ deux fois plus importante.

- Le séchage à une température de $80^{\circ} \mathrm{C}$ (Stade III) soit immédiatement après la récolte $\left(\mathrm{R}_{\mathbf{8 0}}\right)$ soit après les traitements d'attente $\left(\mathrm{F}_{\mathbf{8 0}}, \mathrm{F}_{80}\right)$ est sans effet significatif, la diminution de rendement pour ces deux derniers échantillons restant du même ordre que celles constatées au stade II. Ce résultat est à souligner car les essais effectués sur des maïs séchés dans les mêmes conditions mais au laboratoire ont fait apparaître une diminution de rendement voisine de $5 \mathrm{p}$. Ioo.

- Le séchage à une température de $\mathrm{I}_{4}{ }^{\circ} \mathrm{C}$ (Stade III) provoque une baisse de rendement générale et importante : I6,6 p. Ioo pour $\mathrm{R}_{\mathbf{1 4 0}}$, I9,8 $\mathrm{p}$. Ioo pour $\mathrm{E}_{\mathbf{1 4 0}}$ et Io p. Ioo pour $F_{140}$. Toutefois l'effet dépressif du seul séchage est plus grand s'il intervient dès la récolte $\left(R_{140}\right.$, stade III) que s'il est réalisé après les traitements de préstockage $\left(\mathrm{E}_{140}, \mathrm{~F}_{140}\right.$, stade III). Ceci peut s'expliquer par le fait que les humidités initiales du maîs et le rythme du séchage étaient différents dans les trois cas ou par des modifications de l'état physiologique des grains au cours du préstockage. 
RENDEMENT EN AMIDON DU MAIIS

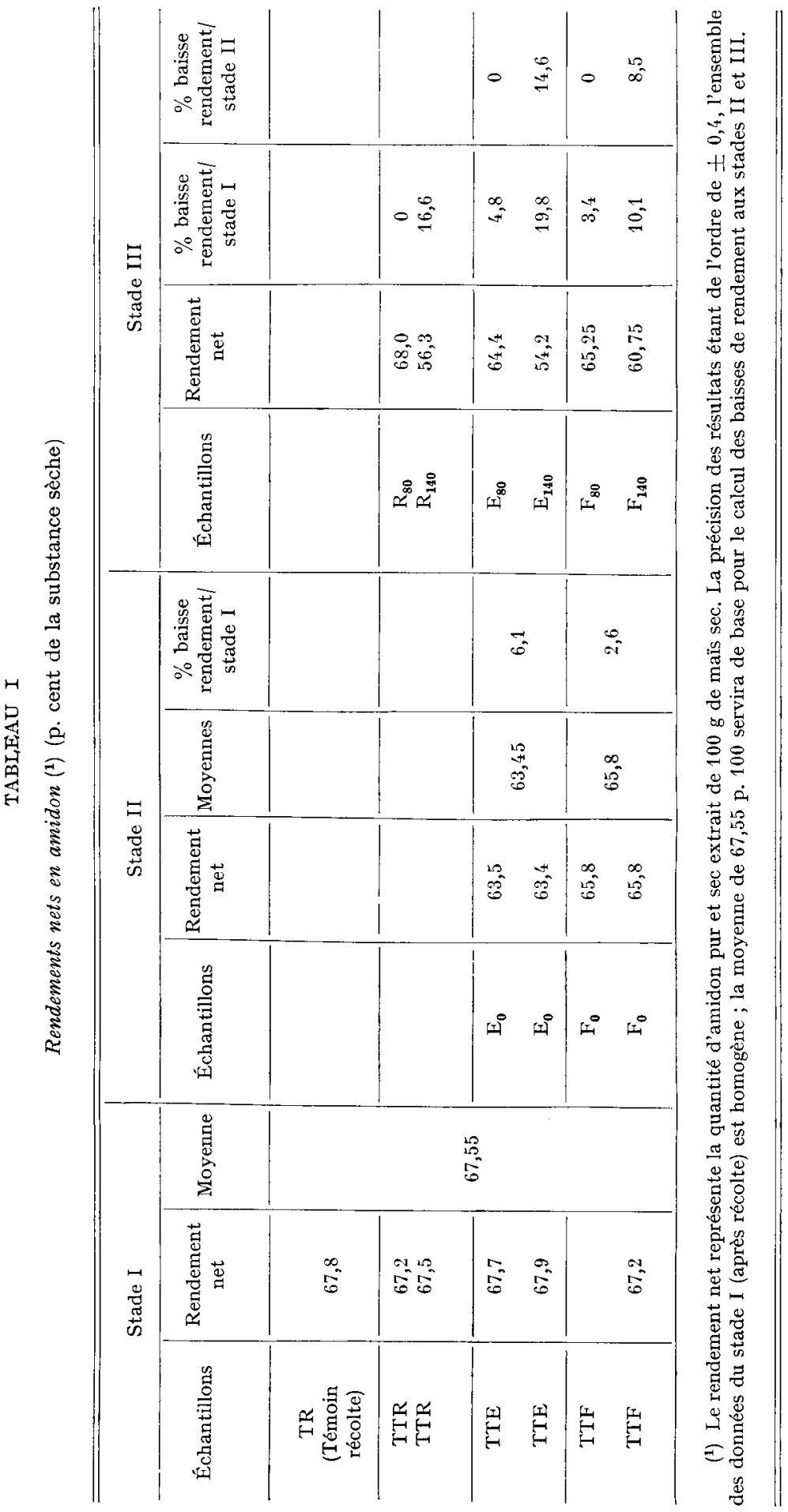


En résumé, le séchage industriel du maïs à une température de $80^{\circ} \mathrm{C}$ ne modifie pas le rendement en amidon du moins dans les conditions de l'essai, par contre, 1'utilisation d'air à $I 40^{\circ} \mathrm{C}$ a pour conséquence un effet dépressif plus important imputable à la dénaturation des protéines de l'endosperme et à l'éclatement des grains d'amidon (SAINT-LìiBE, I969).

D'après les analyses commerciales (ScoTTI, II) seuls les maïs TR et $R_{80}$ sont "sains, loyaux et marchands" (qualité type) ; sur le plan de la valeur amidonnière il en est de même, mais la classification commerciale ne peut donner un gradient de qualité comme l'étude du taux d'extraction. Par ailleurs, il existe une corrélation entre la solubilité des protéines dans 1'alcool (Godon, V) des échantillons séchés à 80 et $140^{\circ} \mathrm{C}$ dès la récolte et leur rendement en amidon (tab1. 2 ). Ce point devra être approfondi, car la détermination de cette caractéristique chimique est beaucoup plus rapide, que celle du rendement en amidon.

\section{2. Étude rhéologique}

Les principaux résultats sont mentionnés dans le tableau 3. On peut en déduire les conclusions suivantes :

- Les deux types de préstockage, “ $\mathrm{E}$ » et “ $\mathrm{F}$ », ne modifient guère les caractéristiques rhéologiques des échantillons $\mathrm{E}_{0}$ et $\mathrm{F}_{0}$ (Stade II) par rapport à celles des témoins du stade I, si ce n'est par une baisse de 1'ordre de Io p. Ioo de la viscosité à $25^{\circ} \mathrm{C}$.

TABLEAU 2

Solubilité des protéines dans l'alcool et rendement en amidon (p. cent de la substance sèche)

\begin{tabular}{l|c|c|c}
\hline \hline & $\begin{array}{l}\text { Témoin } \\
\text { récolte }\end{array}$ & $\mathrm{2}$ & \multicolumn{2}{|c}{ Stade III } \\
\cline { 3 - 4 } & & $\mathrm{R} 80$ & $\mathrm{R} 140$ \\
\hline $\begin{array}{c}\text { Solubilité des protéines } \\
\text { dans l'alcool ......... }\end{array}$ & 24,4 & 22,3 & 11,0 \\
\hline Rendement en amidon ... & 67,8 & 68,0 & 56,3 \\
\hline
\end{tabular}

- Le séchage avec de 1'air à une température de $80^{\circ} \mathrm{C}$ (Stade III) est sans effet sur la température de début de consistance des empois, mais diminue de 1'ordre de 20 p. Ioo la viscosité à $96^{\circ} \mathrm{C}$ et à $25^{\circ} \mathrm{C}$, qu'il y ait eu préstockage $\left(\mathrm{E}_{80}\right.$ et $\left.\mathrm{F}_{80}\right)$ ou non $\left(R_{80}\right)$. Cette diminution est équivalente à celle enregistrée après une irradiation gamma à la dose de I5o krad du maîs à $38 \mathrm{p}$. Ioo d'humidité, ou à celle mise en évidence après l'irradiation d'amidon industriel à la dose de $300 \mathrm{krad}$.

- Le séchage avec de 1'air à une température de $140^{\circ} \mathrm{C}$ (Stade III) induit des modifications beaucoup plus importantes, diminution de la viscosité à $96^{\circ} \mathrm{C}$ et $25^{\circ} \mathrm{C}$ de $1^{\prime}$ 'ordre de $5^{\circ} \mathrm{p}$. Ioo et augmentation de 2 à $4^{\circ} \mathrm{C}$ de la température de début de consistance des empois. Comme pour le taux d'extraction d'amidon, l'effet du séchage est plus important lorsqu'il intervient dès la récolte $\left(\mathbf{R}_{\mathbf{1 4 0}}\right)$ que lorsqu'il intervient après une phase de préstockage $\left(\mathrm{E}_{140}, \mathrm{~F}_{140}\right)$. 
RENDEMENT EN AMIDON DU MAÏS

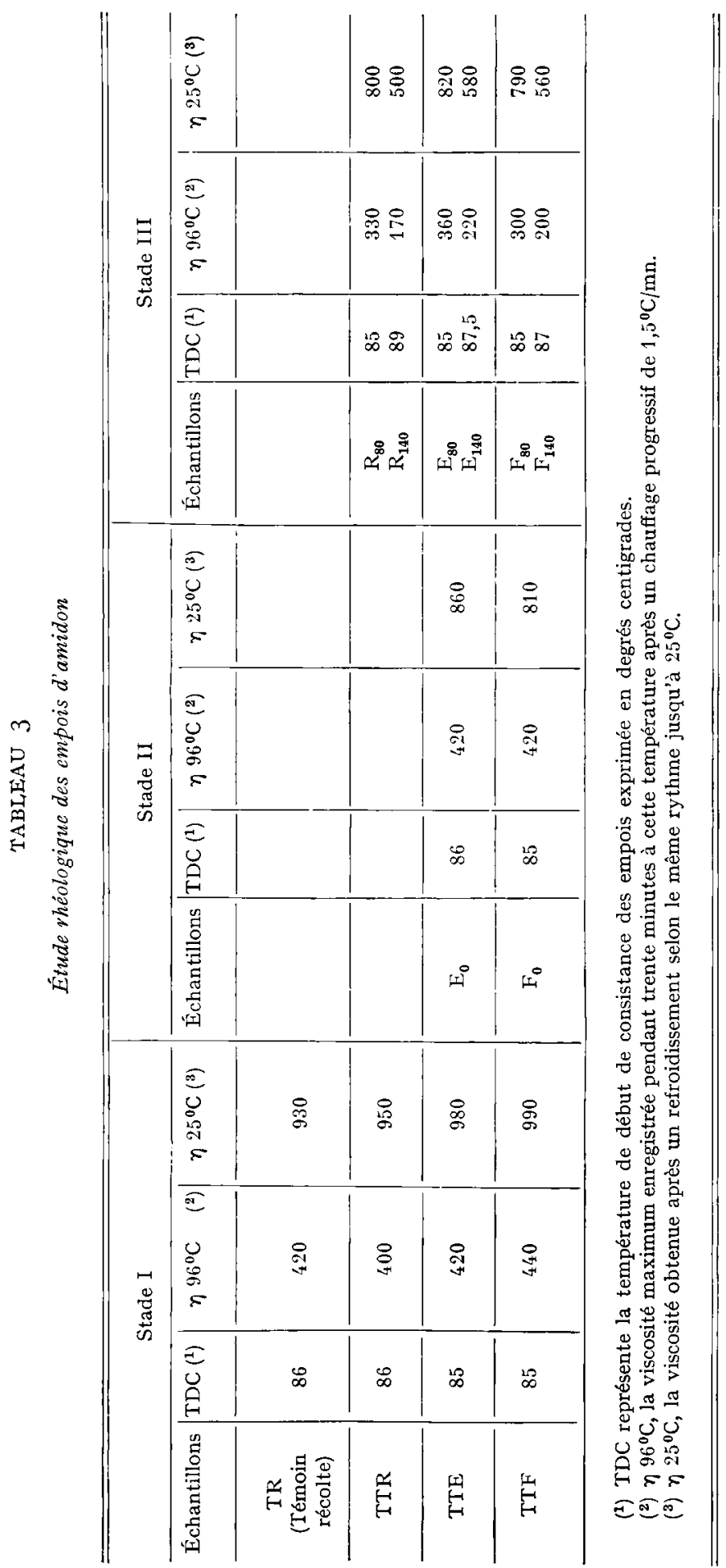


La formation d'un empois à partir d'amidon extrait de mais séché avec del'air chaud paraît d'autant plus difficile que la température de séchage est plus élevée, tout se passant comme si la pénétration de l'eau et l'affinité de l'eau étaient moins bonnes. Il en est de même au cours d'un traitement hydrothermique del'amidon (Schierbaum, I966) dont le mode d'action est comparable à celui du séchage du maïs avec de l'air chaud.

\section{CONCLUSION}

Les analyses commerciales ne semblent pas être suffisamment sensibles pour apprécier les variations de qualité du mais destiné à l'amidonnerie (traitement ( $\mathrm{E}$ ) et « $\mathrm{F} 川$ ). Néanmoins, le séchage industriel avec de l'air à $80^{\circ} \mathrm{C}$, pratiqué immédiatement après récolte $\left(\mathrm{R}_{80}\right)$, permet d'obtenir dans les conditions de l'expérience un maïs d'une part " sain, loyal et marchand ", d'autre part, de bonne qualité pour l'amidonnerie. Par contre, le grain est profondément altéré et 1'amidon dégradé lorsque la température atteint $140^{\circ} \mathrm{C}$; le maïs obtenu dans ces conditions constitue une matière première de mauvaise qualité pour l'amidonnerie. Toutefois, avant de porter un jugement définitif il faudra entreprendre une étude complète sur l'influence respective de la température de l'air et du rythme du séchage en fonction de la variété, de la maturité, de l'humidité initiale et finale du grain.

\section{SUMMARY}

\section{VI. - QUALITY OF KERNELS FOR THE STARCH INDUSTRY}

The quality of processing for the starch industry has been measured by the technological yield in starch estimated in a pilot factory and by the measurement of starch rheology.

In the different samples, maize air dried at $80^{\circ} \mathrm{C}$ has the same starch yield as the control air-dried corn, the rheological characteristics have shown no marked variations. On the other hand, the kernels dried at $14 \mathrm{O}^{\circ} \mathrm{C}$ are considerably damaged, especially with regard to the starch fraction. It may be considered that this kind of grain is a poor raw material for the starch industry.

\section{RÉFÉRENCES BIBLIOGRAPHIQUES}

Association pour la Promotion Indústrie-Agriculture (A. P. R. I. A., Paris), ig68. Le maïs et ses industries. Apria éd. Paris, $215 \mathrm{p}$.

Gausman H. W., Ramser J. H., Dungan G. H., Farle F. R., Mac Masters M, M., Hall H. H. et BAIRD P. D., I952. Some effects of artificial drying of corn grain. Plant Physiol., 27, 794-802.

Mac Masters M. M., Earle F. R., Hall H. H., Ramser J. H. et Dungan G. H., I954. Studies on the effect of drying conditions upon the composition and suitability for wet milling of artificially dried corn. Cereal Chem., 31, 45 I-461.

Mac Masters M. M., Frwkner M. D., Holzapfel M. M., Ramser J. H. et Dungan G. H., I959. A study of the effect of drying conditions on the suitability for starch production of corn artificially dried after shelling. Cereal Chem., 36, 247-260.

SAInt-Lìbe L., I969. Effets de l'irradiation $\lambda$ sur quelques caractéristiques technologiques du maïs d'amidonnerie. Die Stärke, 21, 8-I3.

Saint-Lèbe L., Jossoud M., André C., I965. Extraction de l'amidon de maïs. Die Stärke, 17, $34 \mathrm{I}-346$. 
Schierbaum F., 1966. Zur Veränderlichkeit der physikalischen und chemischen Eigenschaften der Kartoffelstärke. Die Stärke, 18, I ro-r 15.

WAHL G., I966. a) Maistrocknung und Nassmüllerei. I. Mitteilung : Untersuchung der Verarbeitungsfähigkeit von künstlich getrocknetem Mais. Die Stärke, 18, I r-r9.

WAKL G., Ig66. b) Maistrocknung und Nassmüllerei. 2. Mitteilung : Uber die Kolloid-Chemischen Eigenschaften der aus künstlich getrocknetem Mais gewonnenen Stärke. Die Stärke, 18, 383-39o.

Watson S. A., Hirata Y., r962. Some wet-milling properties of artificially dried corn. Cereal Chem., $39,35-44$. 